\title{
Characterization and rheological study of the galactomannan extracted from seeds of Cassia grandis
}

\author{
Priscilla B.S. Albuquerque a, Wilson Barros Jr. ${ }^{\mathrm{b}}$, Gustavo R.C. Santos ${ }^{\mathrm{c}}$, Maria T.S. Correia ${ }^{\mathrm{a}}$, \\ Paulo A.S. Mourão ${ }^{c}$, José A. Teixeira ${ }^{d}$, Maria G. Carneiro-da-Cunha ${ }^{\mathrm{a}, *}$ \\ a Departamento de Bioquímica/Laboratório de Imunopatologia Keizo Asami-LIKA, Universidade Federal de Pernambuco, Avenue Prof. Moraes Rego s/n, \\ Recife CEP: 50.670-420, PE, Brazil \\ b Departamento de Física, Universidade Federal de Pernambuco, Avenue prof. Luiz Freire s/n, Recife CEP 50670-901, PE, Brazil \\ ' Laboratório de Tecido Conjuntivo, Hospital Universitário Clementino Fraga Filho and Programa de Glicobiologia, Instituto de Bioquímica Médica, \\ Universidade Federal do Rio de Janeiro, Rio de Janeiro, Brazil \\ d IBB-Institute for Biotechnology and Bioengineering, Centre of Biological Engineering, Universidade do Minho, Campus de Gualtar, Braga 4710-057, \\ Portugal
}

\section{A R T I C L E I N F O}

\section{Article history:}

Received 19 October 2013

Received in revised form

10 December 2013

Accepted 5 January 2014

Available online 10 January 2014

\section{Keywords:}

Galactomannan

Cassia grandis

Rheology

X-ray

NMR

Characterization.

\begin{abstract}
A B S T R A C T
Galactomannan extracted from seeds of Cassia grandis with $0.1 \mathrm{M} \mathrm{NaCl}$, followed by ethanol precipitation, presented a yield of $36 \pm 8 \%$. The polysaccharide has a constant mannose/galactose ratio (2.44:1). Methylation analysis, one and two dimensional NMR spectroscopy confirmed that the polysaccharide has a central core composed of 4-linked $\beta$-mannose units, with branches of galactose, linked to the carbohydrate core through $\alpha(1-6)$ linkage. The amorphous nature of the galactomannan was confirmed by X-ray diffraction. Rheological characterization exhibited Newtonian plateaus followed by shear-thinning zones characteristic of polymer solutions up to $1.5 \%(\mathrm{w} / \mathrm{v})$ and above this value the system exhibited yield stress associated with a weak gel. Adjusting stress-strain curves confirmed a $1.6 \%(\mathrm{w} / \mathrm{v})$ as the galactomannan concentration value for the sol-gel transition. These results indicate that the galactomannan extracted from $C$. grandis seeds presents rheological characteristics suitable for applications in pharmaceutical, biomedical, cosmetic and food industries.
\end{abstract}

(c) 2014 Elsevier Ltd. All rights reserved.

\section{Introduction}

Galactomannans are polysaccharides widespread in nature. Their basic structure is formed by a central core of $\beta(1 \rightarrow 4)$ linked D-mannopyranose(Man) to which $\alpha(1 \rightarrow 6)$-linked $\alpha$-Dgalactopyranosyl(Gal) units are attached. These polysaccharides are commonly extracted from the endosperm of numerous seed plants (particularly the Leguminosae) where they develop energy-reserve and hydration functions. Due to its specific physicochemical properties such as high molecular weight, water solubility, non-ionic character, and absence of toxicity (Gidley \& Reid, 2006; Pollard, Eder, Fischer, \& Windhab, 2010), this polysaccharide can be used in food, pharmaceutical, biomedical, cosmetic, textile and paper industries (Srivastava \& Kapoor, 2005), especially as an emulsion stabilizer, in the preparation of films (Cerqueira et al., 2009a, 2011; Mikkonen et al., 2007) and as a gelling agent

\footnotetext{
* Corresponding author. Tel.: +55 812126 8540; fax: +55 8121268576

E-mail address: mgcc@ufpe.br (M.G. Carneiro-da-Cunha).
}

(Pinheiro et al., 2011; Vendruscolo, Andreazza, Ganter, Ferrero, \& Bresolin, 2005).

Galactomannans are used in various pharmaceutical forms, such as tablets or capsules, hydrogels and films. Besides the simple use as an inert excipient, this polysaccharide plays a role in controlled drug delivery, especially in colonic environment, being used as a matrix or coating material (Silveira \& Bresolin, 2011). In the food industry, galactomannans from non-traditional sources are being evaluated as films or coatings to prolong the shelf life of various foods such as tropical fruits (Cerqueira et al., 2009a) and ricotta cheese (Martins, Cerqueira, Souza, Avides, \& Vicente, 2010).

Galactomannans extracted from seeds of four different species of Leguminosae, Adenanthera pavonina, Caesalpinia pulcherrima, Gleditsia triacanthos and Sophora japonica, showed adequate characteristics for food and biomedical industry applications (Cerqueira et al., 2009b). The synergistic interactions of two non-conventional galactomannans (G. triacanthos and S. Japonica) with carrageenan and xanthan represent alternatives to the traditional galactomannans guar gum and locust bean gum with the same polysaccharides, and can be used to provide different rheological characteristics of food products (Pinheiro et al., 2011). The content of water soluble gum (32-34\%) found in the seeds of Cassia grandis from India 
was reported by Joshi and Kapoor (2003) as having characteristics to become a potential source of this polysaccharide. New sources of seed gums are demanded worldwide and searching for renewable sources of these polysaccharides is justified. In spite of the rich biodiversity of the local flora and of the favorable climate for their production, Latin American sources of galactomannans are not yet well known.

C. grandis is a small to medium size tree, belonging to the Leguminosae family and subfamily of Caesalpinioideae, native of tropical and Central America and West India and widespread in Brazil, a tropical country. As $C$. grandis seeds contain large amounts of galactomannan, this work aimed at the extraction, structural and rheological characterization of this polysaccharide for future biotechnological applications.

\section{Material and methods}

\subsection{Material}

The pods of $C$. grandis were collected at the rural zone of Pernambuco State, in the city of Angelim (Brazil), in July 2011. Ethanol 99.8\%, acetone PA, sodium chloride and phenol were obtained from Vetec Fine Chemicals Ltda. (Brazil). All other chemicals were of analytical grade.

\subsection{Extraction of the galactomannan from $C$. grandis seeds}

The pods of $C$. grandis were immersed in distilled water at $25^{\circ} \mathrm{C}$ for $18 \mathrm{~h}$ and then separated in a half part, revealing the seeds trapped in a black and stick mass. The seeds were removed and placed in an oven at $100^{\circ} \mathrm{C}$ until reaching a constant weight. The dry seeds $\left(M_{\mathrm{i}}\right)$ were boiled in distilled water $[1: 5(\mathrm{w} / \mathrm{v})]$ at $100^{\circ} \mathrm{C}$ for $1 \mathrm{~h}$ for enzyme inactivation and maintained in water by $18 \mathrm{~h}$ at $25^{\circ} \mathrm{C}$ to facilitate removal of the hull. After this period, the hull was removed and the seeds without hull, endosperm plus germ, were triturated in a blender with $0.1 \mathrm{M} \mathrm{NaCl}[5 \%(\mathrm{w} / \mathrm{v})]$ at $25^{\circ} \mathrm{C}$, filtered through a vual tissue, that was followed by a new filtration using screen printing cloth (90 thread type) and precipitated with $46 \%$ ethanol [ $1: 3(\mathrm{v} / \mathrm{v})$ ] for $18 \mathrm{~h}$. The white precipitate obtained was filtered on screen printing cloth (110 thread type), washed with $100 \%$ ethanol $[1: 3(\mathrm{w} / \mathrm{v})]$ for $30 \mathrm{~min}$ and two times with acetone PA [1:3 (w/v)] for $30 \mathrm{~min}$, filtered on screen printing cloth (110 thread type) between each washing. The precipitated galactomannan obtained was placed in an oven at $100^{\circ} \mathrm{C}$ until constant weight $\left(M_{\mathrm{f}}\right)$ and finally pulverized and kept in a dry place until further use.The yield of extraction was calculated by dividing the final mass of galactomannan $\left(M_{\mathrm{f}}\right)$ by the initial mass of the dry seeds $\left(M_{\mathrm{i}}\right)$ and express as \% $\mathrm{w} / \mathrm{w}$. The experiment was carried out in triplicate and the results expressed as mean \pm standard deviation.Structural characterization

\subsubsection{Fourier Transform Infrared Spectroscopy and X ray patterns}

The polysaccharide extracted from $C$. grandis seeds was characterized by Fourier Transform Infrared (FTIR) Spectroscopy on a VERTEX 70 (Bruker Optics, USA) spectrometer under dry air at room temperature $\left(25^{\circ} \mathrm{C}\right)$ using $\mathrm{KBr}$ pellets. The polysaccharide was mixed with $\mathrm{KBr}$ then compressed into $1 \mathrm{~mm}$ tablets and spectra were scanned between 4000 and $500 \mathrm{~cm}^{-1}$ with $4 \mathrm{~cm}^{-1}$ of resolution.

The crystal structure of the polysaccharide was analyzed by $\mathrm{X}$ ray diffraction using a Bruker D8 Advance (Germany) diffractometer $(30 \mathrm{kV}, 30 \mathrm{~mA})$ equipped with $\mathrm{Cu} K_{\alpha}$ radiation at wavelength of $0.154 \mathrm{~nm}$. The measurements were carried out for an angular interval varying from $5-60^{\circ}$ ( $2 \theta$ range), scanning rate of $5 \%$ min, step of $0.02^{\circ}$ and 2 s per step. To determine the polysaccharide crystallinity, the total diffracted area and the area under the crystallinity peaks were evaluated by integration after correcting the data for absorption. The ratio of the crystalline area to the total area was taken as the relative crystallinity.

\subsubsection{Monosaccharide composition and methylation analysis of the polysaccharide}

The polysaccharide $(5 \mathrm{mg}$ ) was hydrolyzed with $5 \mathrm{M}$ trifluoroacetic acid for $4 \mathrm{~h}$ at $100^{\circ} \mathrm{C}$, reduced with borohydride, and the alditols were acetylated with acetic anhydride:pyridine $(1: 1$, $\mathrm{v} / \mathrm{v})$. The acetylated alditols were dissolved in chloroform and analyzed in a gas-liquid chromatography/mass spectrometry (GCMS-QP2010 Shimadzu, Japan) with a Restek column RTX-5MS, according to Kircher (1960). For methylation analysis the polysaccharide ( $5 \mathrm{mg}$ ) was subjected to two rounds of methylation as described by Ciucanu and Kerek (1984). The methylated polysaccharide was hydrolyzed, reduced with borohydride, acetylated and analyzed on a GCMS instrument, as described above.

\subsubsection{Nuclear magnetic resonance spectroscopy}

One (1D) and two-dimensional (2D) spectra of the polysaccharide were recorded using a Bruker DRX $600 \mathrm{MHz}$ apparatus with a triple resonance probe, as described previously (Tovar et al., 2012). Approximately $5 \mathrm{mg}$ of each sample was dissolved in $0.5 \mathrm{ml}$ of $99.9 \%$ deuterium oxide (Cambridge Isotope Laboratory, Cambridge, MA, USA). All spectra were recorded at $45^{\circ} \mathrm{C}$ with HOD (deuterated water exhibiting a peak due to exchange with residual $\mathrm{H}_{2} \mathrm{O}$ ) suppression by presaturation. For $1 \mathrm{D}^{1} \mathrm{H}$ NMR spectra, 32 scans were recorded, using an inter-scan delay equals $1 \mathrm{~s}$. For $2 \mathrm{D}$ ${ }^{1} \mathrm{H} /{ }^{1} \mathrm{H}$ TOCSY (total correlated spectroscopy) and ${ }^{1} \mathrm{H} /{ }^{13} \mathrm{C} \mathrm{HSQC}$ (heteronuclear single quantum coherence) experiments, spectra were recorded using states TPPI (time proportion phase incrementation) for quadrature detection in the indirect dimension. TOCSY spectra were run with $4046 \times 400$ points with a spinlock field of $10 \mathrm{kHz}$ and a mixing time of $80 \mathrm{~ms}$. Two-dimensional ${ }^{1} \mathrm{H} /{ }^{13} \mathrm{C}$ multiplicityedited HSQC spectra were recorded at $45^{\circ} \mathrm{C}$ with HOD suppression by presaturation, with 256 scans. The increment number setup was set to 64 , and states-TPPI were used for quadrature detection in the indirect dimension and run with $1024 \times 256$ points with globally optimized alternating phase rectangular pulses (GARP) for decoupling. Chemical shifts were displayed relative to external trimethyl-silylpropionic acid at $0 \mathrm{ppm}$ for ${ }^{1} \mathrm{H}$ and relative to methanol for ${ }^{13} \mathrm{C}$.

\subsection{Rheological measurements}

The experiments were conducted in a stress controlled rheometer (Anton Paar MCR 301) equipped with a temperature controller.

The less viscous samples [0.5-1.0\% $(\mathrm{w} / \mathrm{v})]$ were inserted into a two concentric cylinder geometry cell with outer diameter (o.d.) $=28 \mathrm{~mm}$ and internal diameter (i.d.) $=24 \mathrm{~mm}$. For the more viscous samples [1.5 and $2 \%(\mathrm{w} / \mathrm{v})$ ] a circular parallel plate cell with internal diameter (i.d.) $=25 \mathrm{~mm}$ and a height $(h)=1 \mathrm{~mm}$ gap between plates was employed. Unless when explicitly stated, all rheology experiments were conducted at $25^{\circ} \mathrm{C}$.

\subsubsection{Rotational flow studies}

The flow studies were conducted in continuous steady state shear rotational mode. The shear-rate, $\dot{\gamma}$, externally applied in the sample varied from 1 to $1000 \mathrm{~s}^{-1}$ and 50 points were acquired. The apparent viscosity, $\eta$, response was recorded.

\subsubsection{Oscillatory flow studies}

The dynamic oscillatory mode studies were conducted varying the applied torque/deformation angular frequency, $\omega$, from 1 to $500 \mathrm{rad} \mathrm{s}^{-1}$. The storage $\left(G^{\prime}\right)$ and loss $\left(G^{\prime \prime}\right)$ module sample responses 
were recorded during the frequency sweep at a rate of one acquisition every $5 \mathrm{~s}$. The strain deformation amplitude, $\gamma$, was fixed at $0.2 \%$ amplitude and 50 points were acquired.

\subsubsection{Temperature stability studies}

For the temperature stability study, the rheometer was operated in oscillatory mode. For a fixed frequency $\omega=2 \pi \mathrm{rad} \mathrm{s}^{-1}$ and an externally fixed applied shear stress, $\tau=2 \mathrm{~Pa}$, the temperature was ramped up continuously at a rate of $5^{\circ} \mathrm{C}$ per min and the storage $\left(G^{\prime}\right)$ and loss $\left(G^{\prime \prime}\right)$ module sample responses were recorded. The temperature interval was from $0{ }^{\circ} \mathrm{C}$ to $70^{\circ} \mathrm{C}$. Data was recorded every $6 s$ and 241 points were collected.

\subsubsection{Stress-strain studies}

For the stress-strain study, the rheometer was operated in oscillatory mode. For a fixed frequency $\omega=2 \pi-\mathrm{rad} \mathrm{s}^{-1}$ an externally applied shear stress, $\tau$, was varied from 1 to $300 \mathrm{~Pa}$ and the storage $\left(G^{\prime}\right)$ and loss $\left(G^{\prime \prime}\right)$ module sample responses were recorded. One acquisition was performed every $5 \mathrm{~s}$ and 50 points were obtained.

\section{Results and discussion}

\subsection{Extraction and yield}

In most of the studies involving extraction of galactomannans from legumes endosperm, milling and extraction procedures are not specified in detail or even mentioned. In general, the seed treatment includes sequential milling of the whole seeds and aqueous extraction of the galactomannans by several agitation steps and subsequent precipitation in alcoholic medium (Dakia Wathelet, \& Paquot, 2007; Pollard et al., 2008).

The yield of polysaccharide extraction from seeds is generally expressed as a percentage of dry mass obtained after extraction in relation with the dry weight of seeds (Buckeridge, Panegassi, Rocha, \& Dietrich, 1995; Pollard et al., 2010). Many of the seed gums marketed are constituted of endosperm plus germ obtained only with hull removal (Perduca et al., 2013).

Considering the importance of the purity of the final product as well as the need to reduce the number of unit operations involved in the steps of extraction and purification, especially at industrial level, the samples containing the endosperm plus germ were selected for extraction of galactomannan from $C$. grandis seeds.

The extraction yield was of $36 \pm 8 \%$, similar to $32-34 \%$ found for the seeds of $C$. grandis from India (Joshi \& Kapoor, 2003) and higher than the $26 \%$ found in the seeds of $C$. javanica (Andrade, Azero, Luciano, \& Gonçalves, 1999). Regarding the extraction yield of the galactomannans of the subfamily Caesalpinioideae, several studies report values between 8 and $40 \%$ of the seed weight, which are higher than the values for other species of Brazilian legumes (Dea \& Morrison, 1975; Buckeridge, Panegassi, Rocha, \& Dietrich, 1995). Based on these values, it may be concluded that an efficient methodology was used in this work for the extraction of the galactomannans of the seeds of $C$. grandis.Structural characterization by Fourier Transform Infrared Spectroscopy and X-ray diffraction

The FTIR spectrum confirms that the extracted material is a galactomannan. Absorption at $3389 \mathrm{~cm}^{-1}$ between the regions $3500-3100 \mathrm{~cm}^{-1}$ is characteristic of a carbohydrate ring (Kim, Park, Nam, Lee, \& Lee, 2003; Peng, Zhang, Zeng, \& $\mathrm{Xu}, 2003$ ) and represents hydroxyl stretching vibration of polysaccharides and water involved in hydrogen bonding (Fringant, Tvaroska, Mazeau, Rinaudo, \& Desbrieres, 1995). The absorption at $2928 \mathrm{~cm}^{-1}$ between the regions $3000-2800 \mathrm{~cm}^{-1}$ was assigned to the stretching vibration of the methylene group $(\mathrm{C}-\mathrm{H})$. In the anomeric region $\left(950-700 \mathrm{~cm}^{-1}\right)$, the polysaccharide exhibited the obvious characteristic absorption at 874 and $814 \mathrm{~cm}^{-1}$, assigned to $\alpha$-D-galactopyranose and
$\beta$-D-mannopyranose, respectively (Figueiró, Góes, Moreira, \& Sombra, 2004; Yuen, Choi, Phillips, \& Ma, 2009), revealing the coexistence of $\alpha$ and $\beta$ glycosidic bonds (Kim et al., 2003; Peng et al., 2003).

It is known that polysaccharides naturally interact with water, leading to structural transitions related to amorphous-crystalline transitions, with a great impact on their molecular mobility and functional properties (Yakimets et al., 2007). X ray diffraction (not shown) confirmed the amorphous nature of $C$. grandis galactomannan presenting a broad peak around $2 \theta=20^{\circ}$, characteristic of amorphous structures (Martins et al., 2012).

The crystallinity index, denoted by the alignment of molecules in a particle structure (Mudgil, Barak, \& Khatkar, 2012), was calculated and obtained value of 0.334 that was higher than those obtained from other seed galactomannans, such as $G$. sinensis (0.297), G. melanacantha (0.278) and G. microphylla (0.285) (Jiang, Jian, Cristhian, Zhanga, \& Sun, 2011), as well as for native guar gum (0.240) (Shobha, Vishukumar, Tharanathan, \& Randgaonkar, 2005). The results indicate that the drying process might have improved the organization of the chain thereby increasing the crystallinity of the material (Vendruscolo et al., 2009).

\subsection{Monosaccharide composition}

The monosaccharide composition determined by gas-liquid chromatography/mass spectrometry revealed that the polysaccharide from $C$. grandis seeds is a mixture of $71.0 \%$ mannose and $29.0 \%$ galactose. No other sugar was detected on this polysaccharide up to a limit of $<2.0 \%$ as \% of dry weight. The mannose/galactose ratio $(2.44: 1)$ is similar to the value $(3.15: 1)$ reported for the galactomannan from C. grandis seeds from India (Joshi \& Kapoor, 2003) and also to other Cassia seed galactomannans namely $C$. siamea (2.55:1) (Kapoor, Milas, Taravel, \& Rinaudo, 1996), C. spectabilis (2.6:1) (Kapoor et al., 1998) and C. angustifolia (2.9:1) (Chaubey \& Kapoor, 2001).

Methylation of this polysaccharide showed a preponderance of the 2,3,6-tri-O-methyl derivative from mannose (59\% of the total methylated derivative), indicating that the preponderant structure is composed of 4-linked mannosyl units. Galactose occurs as $2,3,4,6$-tetra- $O$-methyl derivative ( $15 \%$ of total derivative), indicating that this sugar is a non-reducing terminal branches. The detection of 2,3-di-O-methyl mannose (12\%) in approximately the same proportion as the tetra-methyl derivative from galactose, indicates that the branches occur at position 6 of the major mannose backbone. We also detected minor amounts of 2,3,6tri- $O$-methyl (6\% of total methylated derivative) + 2,3-di- $O$-methyl (4\%) derivatives of galactose and unmethylated mannose (4\%). These derivatives may originate from minor structures or incompleted methylation reaction. Methylation analysis confirms that the polysaccharide contains $\sim 75 \%$ mannose and $\sim 25$ galactose, as indicated by the chemical analysis (see above).

The results are altogether different from those previously reported from Bose and Srivastava (1978), for C. grandis seed polysaccharide with respect to sugar composition (Gal,/Man,/Xyl in a ratio of 7:5:1) and structural features, but agrees with that reported from Joshi and Kapoor (2003), for who other Cassia seed galactomannans provides about $2 / 3$ of unbranched mannose units necessary for synergistic interaction, and observed the same three methylated sugars. Other minor derivatives were also detected, which does not decrease the efficiency of the purification process.

\subsection{Nuclear magnetic resonance spectroscopy}

The structure of the galactomannan from $C$. grandis was further investigated using one-dimensional (1D) and two-dimensional (2D) NMR spectroscopy (Figs. 1 and 2). The $1 \mathrm{D}^{1} \mathrm{H}$ NMR spectrum 


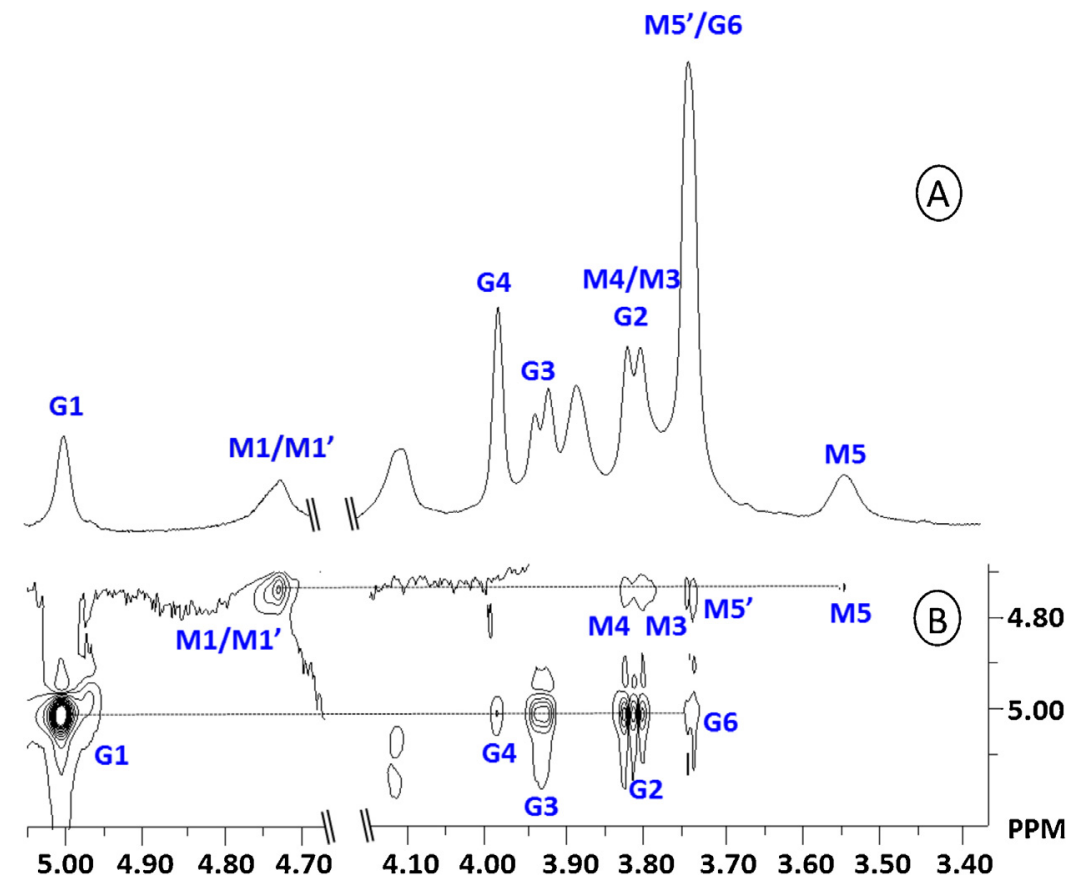

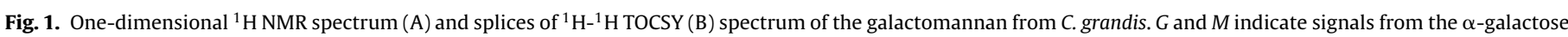
and $\beta$-mannose units, respectively.

Table 1

${ }^{1} \mathrm{H}$ and ${ }^{13} \mathrm{C}$ chemical shifts of the galactomannan from $C$. grandis.

\begin{tabular}{|c|c|c|c|c|c|c|}
\hline Structure & $\mathrm{H}-1 / \mathrm{C}-1$ & $\mathrm{H}-2 / \mathrm{C}-2$ & $\mathrm{H}-3 / \mathrm{C}-3$ & $\mathrm{H}-4 / \mathrm{C}-4$ & $\mathrm{H}-5 / \mathrm{C}-5$ & $\mathrm{H}-6 / \mathrm{C}-6$ \\
\hline$\alpha-D-G a l$ & $5.01 / 99.61$ & $3.81 / 69.24$ & $3.93 / 70.19$ & $3.99 / 70.12$ & $3.89 / 72.10$ & $3.74 / 61.97$ \\
\hline$\beta$-D-Man unbranched at 0-6 & $4.73 / 100.96$ & $4.11 / 70.74$ & $3.80 / 72.23$ & $3.81 / 77.37$ & $3.55 / 75.86$ & $3.90 / 61.34$ \\
\hline$\beta$-D-Man branched at 0-6 & $4.74 / 101.15$ & $4.11 / 70.74$ & $3.80 / 72.23$ & $3.83 / 77.64$ & $3.75 / 74.20$ & $3.96 / 67.37$ \\
\hline
\end{tabular}

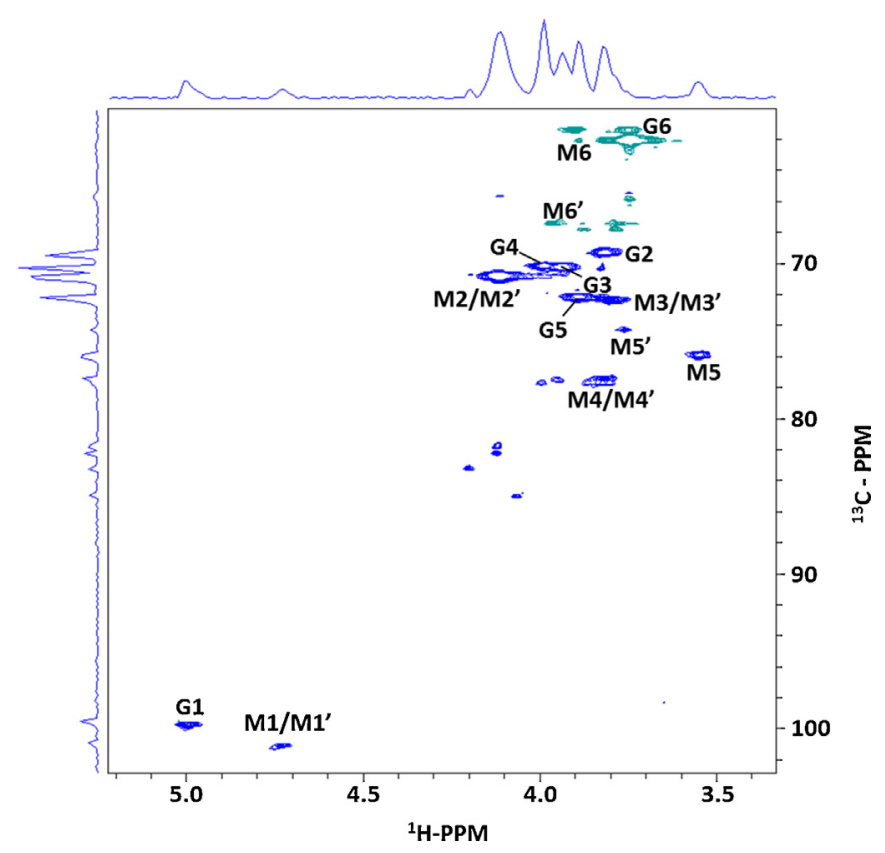

Fig. 2. ${ }^{1} \mathrm{H}^{-13} \mathrm{C}$ HSQC spectrum of the galactomannan from $C$. grandis. The signals of $\mathrm{CH}$ carbon/proton are in blue (phase signals) and those from $\mathrm{CH}_{2}$ in green (antiphase). $G$ and $M$ indicate signals from the $\alpha$-galactose and $\beta$-mannose units, respectively.
(Fig. 1A) clearly showed a ${ }^{1} \mathrm{H}$ anomeric signal of $\alpha$-galactose at $5.01 \mathrm{ppm}$. The anomeric proton from $\beta$-mannose resonates in the same region as the water signal. After we increased the temperature from $35^{\circ} \mathrm{C}$ to $45^{\circ} \mathrm{C}$ we were able to identify this signal at $4.73 \mathrm{ppm}$ but its intensity still affected by the program use to suppress the water signal. As a consequence, the signal of the anomeric protons from the $\beta$-mannose units is underestimated when compared with the chemical and methylation analysis (see above).

The ${ }^{1} \mathrm{H}_{-}{ }^{1} \mathrm{H}$ TOCSY spectrum (Fig. 1B) allowed us to identify the major spin systems. In particular we confirm that the non-terminal branch unit is $\alpha$-galactose. The polysaccharide backbone contains $\beta$-mannose residues as substituted and non-substituted units. The $\mathrm{H}-1, \mathrm{H}-2$ and $\mathrm{H}-3$ chemical shifts of these two units are coincident (Table 1).

The edited ${ }^{1} \mathrm{H}_{-}{ }^{13} \mathrm{C}$ HSQC spectrum (Fig. 2) allowed us to distinguish some of these two $\beta$-mannose units. In this spectrum the signals from $\mathrm{H} 6$ occurs as anti-phase signals (shown in green in the panel). Clearly the ${ }^{13} \mathrm{C}$ signal from 6 -branched $\beta$-mannose unit is down filed shifted, as expected for a glycosylated position. The values of ${ }^{1} \mathrm{H}$ and ${ }^{13} \mathrm{C}$ chemical shifts are shown in Table 1 and coincident with the structure proposed for a polysaccharide composed of 4-linked $\beta$-mannose units, with branches of galactose, linked to the carbohydrate core through $\alpha(1-6)$ linkage.

\subsection{Rheological characterization}

\subsubsection{Flow results}

Fig. 3 depicts flow experiments where the apparent viscosity of galactomannan (gal.) water solutions is measured as a function of 


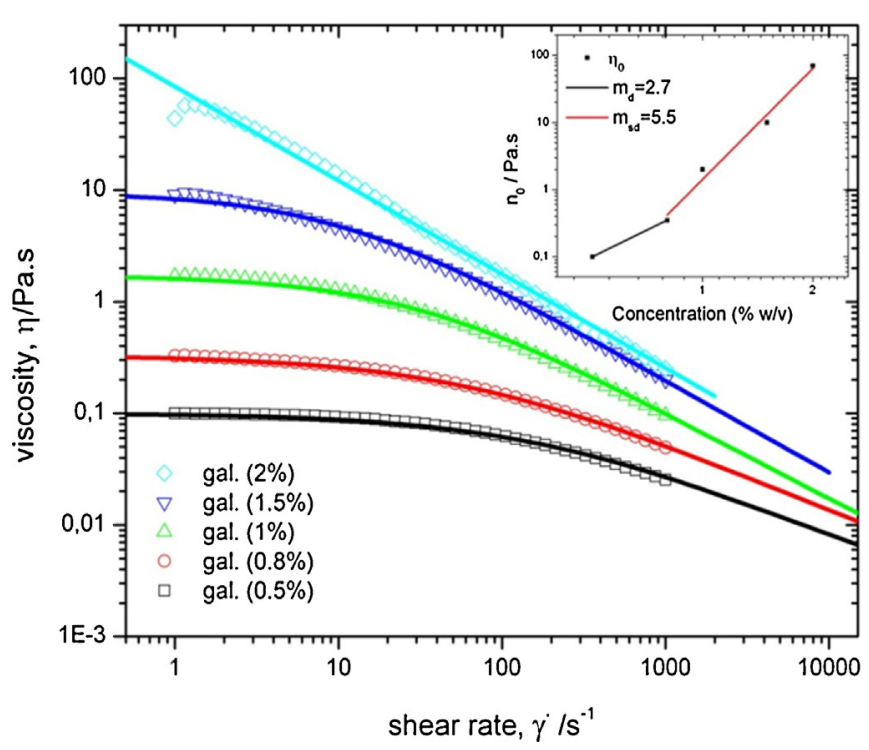

Fig. 3. Rotational steady flow experiments measurements of apparent viscosity $\eta$ as a function of shear-rate $\dot{\gamma}$. The inset shows the zero-shear viscosity, $\eta_{0}$, extracted from the main graphic, as a function of galactomannan concentration.

shear-rate for different polysaccharide concentrations (w/v). Viscosity shear-thinning was observed for all concentrations tested. However, at small shear-rates and concentrations $<1.0 \%$, the apparent viscosity exhibits a Newtonian plateau. This plateau has its length reduced as the concentration increases finally disappearing completely for $2.0 \%$ concentration.

For concentrations $<1.0 \%$, the Newtonian plateau is followed by a power-law decrease in viscosity as a function of shear rate (shearthinning regime). Within this region, the degree of shear-thinning is quantified by the slope of the viscosity/shear-rate curve in a log-log plot.

The observed shear thinning behavior is in agreement with what has been reported for others galactomannans, such as those extracted from Leucaena leucocephala seeds (Nwokocha \& Williams, 2012) and the most commercially used galactomannan, guar gum (Mudgil, Barak, \& Khatkar, 2012).

The experimental curves depicted in Fig. 3 are a typical example of a polymer solution behavior described by the Cross Model (Cross, 1965). This model, initially devised for dispersion aggregates solutions, has been very successful in describing the shear-thinning behavior of dilute polymer solutions.

The apparent viscosity in a rotational shear flow experiment as a function of shear-rate is given by:

$\eta=\eta_{\infty}+\frac{\eta_{0}-\eta_{\infty}}{1+\left(\frac{\dot{\gamma}}{\dot{\gamma}_{c}}\right)^{p^{\prime}}}$

where $\dot{\gamma}$ is the shear-rate, $\eta_{0}$ is the extrapolated value for the viscosity at zero shear rate, $\eta_{0}=\eta(\dot{\gamma} \rightarrow 0)$, and $\eta_{\infty}$ is the viscosity obtained at infinite shear rate, $\eta_{\infty}=\eta(\dot{\gamma} \rightarrow \infty)$. The latter is expected to be dependent only upon the polymer density, given that contributions from interactions within its microstructure to the viscosity become negligible when compared to the shear energy externally applied in this regime. The parameter $\dot{\gamma}_{c}$ is associated with the rupture of structural linkages; it is the critical shearrate above which shear-thinning behavior sets in. The exponent $p$ describes the degree of shear-thinning and is a value between 0 and 1. Depending on the values obtained for $p$ an associated theoretical model becomes available.

The parameters utilized for fitting the data shown in Fig. 3 (solid lines) are presented on Table 2 . It is clearly shown that the degree
Table 2

Fitting parameters for flow curves of the different galactomannan concentrations depicted in Fig. 3.

\begin{tabular}{lccll}
\hline Galactomannan $[\%(\mathrm{w} / \mathrm{v})]$ & $\eta_{0}(\mathrm{Pas})$ & $\dot{\gamma}_{c}\left(\mathrm{~s}^{-1}\right)$ & $p$ & $\eta_{\infty}(\mathrm{Pa} \mathrm{s})$ \\
\hline 0.5 & 0.10 & 200 & 0.65 & 0.001 \\
0.8 & 0.33 & 70 & 0.65 & 0.001 \\
1.0 & 1.71 & 30 & 0.80 & 0.001 \\
1.5 & 9.43 & 10 & 0.80 & 0.001 \\
2.0 & $70^{*}$ & - & 0.84 & - \\
\hline
\end{tabular}

*This value was an extrapolation to the maximum viscosity value at this concentration.

of shear-thinning (represented by the $p$ value) rises abruptly above $0.8 \%$ concentration. The evaluated shear-rate interval was not sufficient to reach the $\eta_{\infty}$ value. Therefore, in Eq. (1), we have simply used a $\eta_{\infty}=0.001$ Pa s for all concentrations.

In a dilute regime, the degree of shear-thinning, described by the exponent $p$, should be the same for all samples, with the shearthinning curves parallel to each other. Clearly, this is not what happens with our data. Actually, for concentrations up to $0.8 \%$ there is a set of parallel curves $(p \sim 0.6)$ and for concentrations above $0.8 \%$ there is another set of parallel curves $(p \sim 0.8)$. This might indicate a transition from a dilute polymer solution in a separate segments regime (dilute regime) into a more interacting (entangled) arrangement (semi-dilute regime). The value $p=0.84$ is compatible with the chain entangling in monodisperse polymers theory (Graessley, 1967). This model assumes viscosity governed by intermolecular chain entanglements. Therefore the decrease in viscosity with shear rate is caused by shear-induced changes in the network of entanglements.

At low shear rates, the entanglement reconstruction characteristic time is expected to be governed by brownian diffusion effects whereas at higher shear rates the disentangling rate is higher and the system viscosity lowers (Castelain, Doublier, \& Lefebvre, 1987). The change on the degree of shear thinning seems to indicate a concentration dependent phase transition where for concentrations below a threshold the system behaves as individual non interacting strands of polymer (dilute regime) that are stretched along the flow for high shear rates. Above the threshold concentration (semidilute regime), the strands interact topologically and the shear thinning results of the strand stretching and strands disentangling effects, the latter occuring only at higher concentrations. A similar abrupt transition was observed at the same $0.8 \%$ concentration when examining the values of $\eta_{0}$, as a function of concentration (see the top right inset in Fig. 3). The inset shows the zero-shear viscosity, $\eta_{0}$, extracted from the main graphic, as a function of galactomannan concentration. The two regimes depicted were adjusted assuming two regimes of power-law dependence: for the first regime (dilute regime), represented by the black solid line, $\eta_{0} \propto C^{m_{d}}$ with $m_{d}=2.7$; for the second regime (semi-dilute regime), shown by the red solid line $\eta_{0} \propto C^{m_{d}}$ with $m_{d}=5.5$. Since the $2 \%$ concentration did not show a steady value for $\eta_{0}$, the maximum value of viscosity for that concentration was chosen.

From the critical shear-rate data, $\gamma_{\mathrm{c}}$, the shear thinning regime interval is reduced as concentrations decreases. For $0.5 \%$ concentration, the system behaves as a Newtonian fluid for most part of the shear-rate interval. However, at $2.0 \%$ concentration no evidence of a Newtonian plateau is observed indicating that, for the same shear-rate interval, a gel transition has occurred.

\subsubsection{Oscillatory and stress-strain results}

Fig. 4 shows the storage, $G^{\prime}$, and loss, $G^{\prime \prime}$, moduli as a function of different oscillatory parameters. The Fig. $4 \mathrm{~A}$ shows an oscillatory shear-stress experiment where $G^{\prime}$ and $G^{\prime \prime}$ are measured as a function of the oscillatory shear angular frequency, $\omega$, for a small fixed strain amplitude, and Fig. 4B shows a stress-strain 

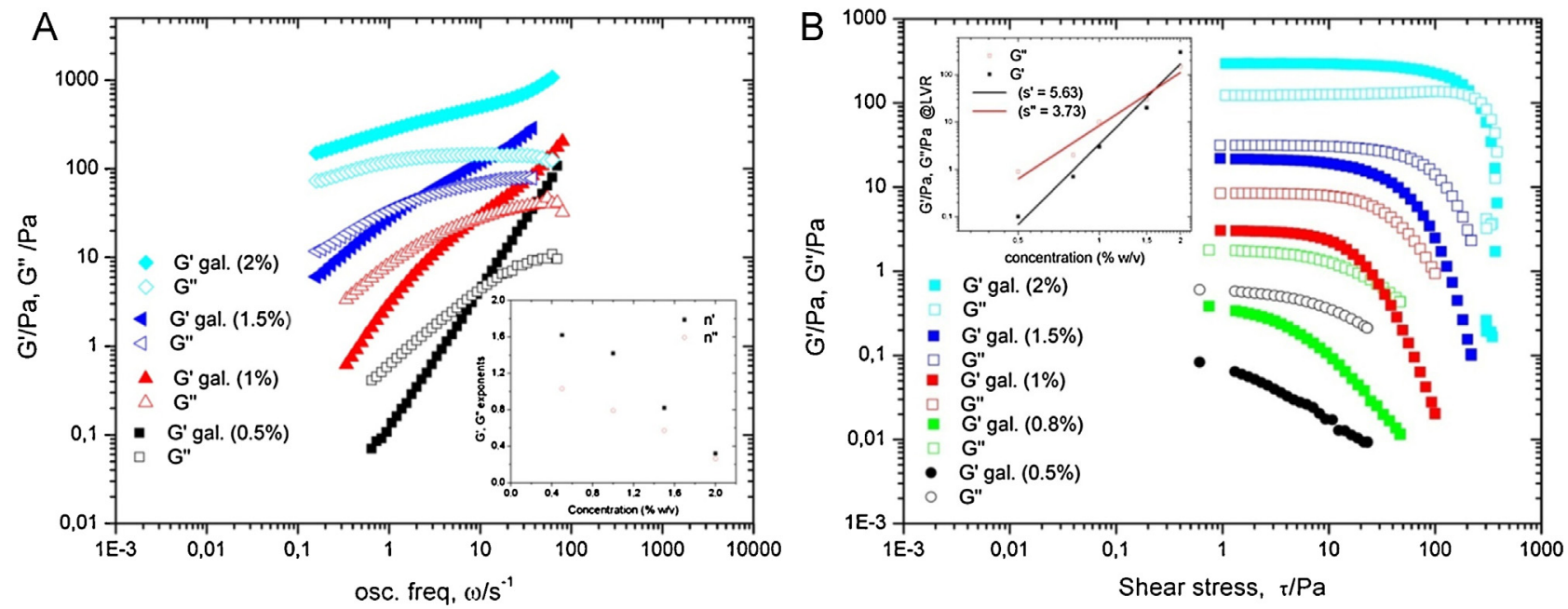

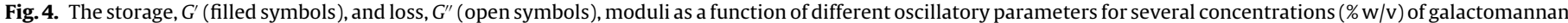
solutions (gal). (A) $G^{\prime}$ and $G^{\prime \prime}$ as a function of an oscillatory stress frequency, $\omega$, and (B) $G^{\prime}$ and $G^{\prime \prime}$ as a function of applied shear stress, $\tau$.

experiment where $G^{\prime}$ and $G^{\prime \prime}$ are measured as a function of an externally applied shear-stress, $\tau$, for several concentrations of galactomannan solutions.

In Fig. 4A the system is kept in the so called linear viscoelastic regime (LVR) where the structure is deformed but not destroyed, offering the opportunity to probe the microstructure of the galactomannan. Both $G^{\prime}$ and $G^{\prime \prime}$ continuously increase as a function of oscillating frequency with their slopes levelling off as the concentration increases, in agreement with the oscillatory behavior observed for other galactomannans (Nwokocha \& Williams, 2012; Perduca et al., 2013).

For concentrations up to $1.5 \%$ there is an observable cross-over between $G^{\prime \prime}$ and $G^{\prime}$. Initially, the galactomannan behaves as a liquid dissipating most of the energy externally applied $\left(G^{\prime \prime}>G^{\prime}\right)$. Above the cross-over, an elastic behavior sets in $\left(G^{\prime}>G^{\prime \prime}\right)$. The cross-over frequency $\omega\left(G^{\prime}=G^{\prime \prime}\right)$ marks the time scale at which the transition from liquid-like to solid-like behavior occurs. The inverse of this frequency can be regarded as a measure of an effective junction lifetime, which increases as the concentration increases. The ability to provide both liquid and solid state features suggests the use of concentrations up to $1.5 \%$ as films/coatings which are widely used in pharmaceutical, biomedical and food industries. Furthermore such a biodegradable source can be used to improve cicatrization processes as well as the existing packaging technology.

For a concentration of $2.0 \%$ the crossover disappears. At this concentration, where the two moduli evolve almost parallel with frequency increase, there is an evidence that the system has reached a gel-like state (Winter \& Chambon, 1986), such as has been observed for xanthan gum (Williams \& Philips, 2000) where there is a tendency to undergo a weak intermolecular chain association for the formation of a three-dimensional structured network behavior (Williams \& Philips, 2000). Although $\left(G^{\prime}>G^{\prime \prime}\right)$, their difference is not sufficient to consider the system as a strong gel. It must also be pointed out that this concentration is the same where the Newtonian plateau disappears (Fig. 3).

The Maxwell model is the simplest model for describing viscoelastic liquid systems. The model considers the behavior of a structured fluid as having a spring (elastic part) and a dash pot (dissipative part) components arranged in series. The equations describing the viscoelastic response of a system as a function of an oscillating stress frequency or strain are:

$G^{\prime}(\omega)=\left(\frac{\eta_{0}}{\omega_{c}}\right) \frac{\omega^{2}}{1+\left(\omega / \omega_{c}\right)^{2}}$

$$
G^{\prime \prime}(\omega)=\frac{\eta_{0} \omega}{1+\left(\omega / \omega_{c}\right)^{2}}
$$

where $\eta_{0}$ is the zero stress viscosity, $\omega$ is the oscillatory stress/strain angular frequency, and $\omega_{\mathrm{c}}$ is the frequency where $G^{\prime \prime}$ and $G^{\prime}$ cross, $\omega\left(G^{\prime}=G^{\prime \prime}\right)$.

Examining both expressions in Eqs. 2 and 3 one derives that at low frequencies, $\omega \ll \omega_{\mathrm{c}}$, the storage and loss moduli behave as $G^{\prime} \sim \omega^{2}$ and $G^{\prime \prime} \sim \omega^{1}$, respectively. This behavior is observed for samples at concentrations below $1.0 \%$. For concentrations above $1.0 \%$ the frequency exponents decrease considerably approaching a common value (see inset in Fig. $4 \mathrm{~A}$ ). In the latter regime, the Maxwell model is not sufficient to explain the viscoelastic behavior. This transition occurs around the same concentration values as in the dilute to semi-dilute transtion in the flow experiments depicted in Fig. 3. The high-frequency regime $\omega \gg \omega_{c}$ was not accessible within the experimental frequency interval utilized.

The exponents $n^{\prime}$, for $G^{\prime} \sim \omega^{n^{\prime}}$; and $n^{\prime \prime}$, for $G^{\prime \prime} \sim \omega^{n^{\prime \prime}}$, are depicted in the inset of Fig. $4 \mathrm{~A}$ as a function of galactomannan concentration. The exponents were obtained by fitting the data in the main graphic considering the frequency interval below each respective cross-over.

These exponents decrease linearly with the increase in concentration, with $n^{\prime}=n^{\prime \prime}$, for high concentrations as expected for gel systems. At lower concentrations, the exponents approach the values predicted by the simple Maxwell model, $n^{\prime}=2$ and $n^{\prime \prime}=1$. At this regime the galactomannan behaves as viscoelastic Maxwell liquids.

In Fig. 4B both moduli increase as a function of concentration with $G^{\prime \prime}>G^{\prime}$ up to $1.5 \%$ for the entire externally applied stress interval. Similar behavior has been observed for the oscillatory data (Fig. 4A). For concentrations of 0.5 and $0.8 \%$, the samples behave as a viscoelastic liquid where the $G^{\prime \prime}$ contribution has a constant value (Newtonian behavior) for a finite stress interval whereas the $G^{\prime}$ contribution decreases as a function of stress with $G^{\prime \prime}>>G^{\prime}$ for the entire stress interval. The system is essentially a Newtonian liquid for moderate applied stresses and cannot sustain elastic linear deformations.

For concentrations of 1.0 and $1.5 \%$, the samples behave again as viscoelastic liquids but now the system can sustain a linear viscoelastic region (LVR) where both $G^{\prime}$ and $G^{\prime \prime}$ are independent of the externally applied stress with $G^{\prime \prime}>G^{\prime \prime}$. This LVR increases as the concentration increases.

For the $2.0 \%$ concentration, the system also exhibits a LVR and, furthermore, $G^{\prime}>G^{\prime \prime}$ over almost the entire shear-stress interval 


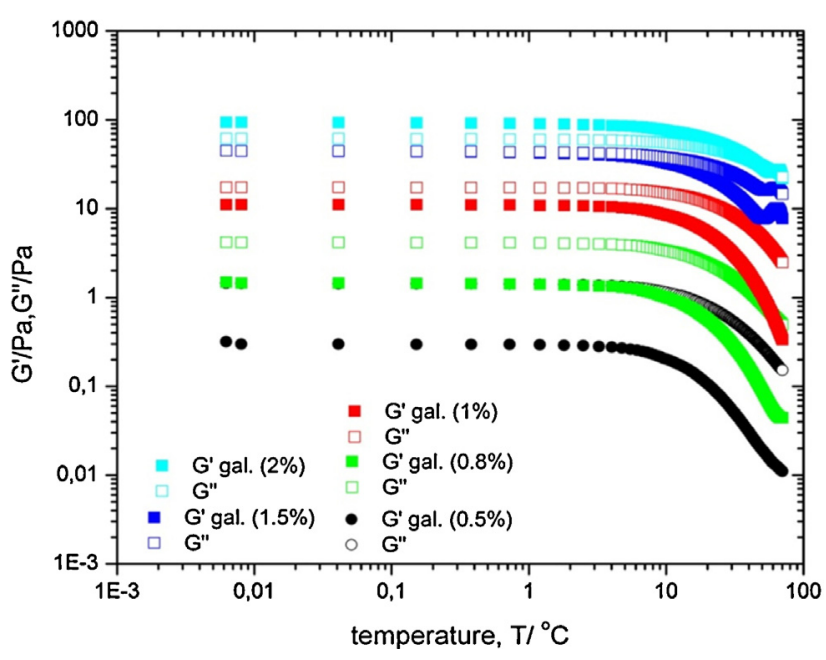

Fig. 5. Temperature stability experiment. The storage modulus, $G^{\prime}$ (filled symbols), and the loss modulus, $G^{\prime \prime}$ (open symbols), are measured as a function of temperature for several concentrations of galactomannan solutions (gal).

evaluated. The low sensitivity to applied shear stress indicates the high stability of the sample at this concentration, behaving as a gel. This caractheristic suggests a promising use of this polymer since, according to other studies, obtaining gels requires mixing different types of galactomannan (Da-Lozzo et al., 2012; Pinheiro et al., 2011; Rinaudo \& Moroni, 2009; Sandolo et al., 2010). Again, this is the same concentration where evidences for gel transition are shown in Figs. 3 and 4A. For larger shear-stress values, there is an observable crossing between the viscoelastic moduli $\left(G^{\prime}=G^{\prime \prime}\right)$. This point is identified as the yield stress of the gel, the point where it starts flowing.

The inset (top left) is a log-log plot constructed by collecting the values of $G^{\prime}$ and $G^{\prime \prime}$ moduli, obtained by extrapolating the points in the LVR $(\tau \rightarrow 0)$ of the main graphic in Fig. $4 \mathrm{~B}$, as a function of the respective galactomannan solution concentration. The change in $G^{\prime \prime}$ within the concentration interval is about 2 orders of magnitude, very similar to the interval of viscosity presented in Fig. 3 for the same concentration interval.

The variation in $G^{\prime}$ is 3 orders of magnitude within the same interval showing that the elastic properties are more sensitive to galactomannan concentration than its viscosity. One can identify in the inset figure a transition from a viscoelastic liquid $\left(G^{\prime \prime}>G^{\prime}\right)$ into a viscoelastic solid $\left(G^{\prime}>G^{\prime \prime}\right)$. This transition occurs at $1.6 \%$ of galactomannan solution concentration and is considered as the point where the polymer solution turns into a gel. Both moduli exhibit a power-law dependence with polymer concentration. The solid lines are fitting functions obtained assuming power-law dependence of $G^{\prime}$ and $G^{\prime \prime}$ with galactomannan concentration obtained exponents were $s^{\prime}=5.63$ for $G^{\prime}$ and $s^{\prime \prime}=3.73$ for $G^{\prime \prime}$ dependence.

\subsubsection{Temperature stability}

Fig. 5 shows a standard temperature stability experiment where the storage and loss moduli are measured as a function of temperature for several concentrations of galactomannan solutions. As in previous experiments, the storage modulus is smaller than the loss modulus up to $1.5 \%$ concentration. Both moduli remain constant (stable) up to $10^{\circ} \mathrm{C}$ for all concentrations and start to change above these temperature. For concentrations above $1.5 \%$ and, more important, for $2.0 \%$ concentration where $G^{\prime}>G^{\prime \prime}$, the moduli variations are considerably small as compared to lower values of concentration for the same temperature interval (from 10 to $70^{\circ} \mathrm{C}$ ).

Many neutral polysaccharides which are rich in hydroxyl groups are able to form tridimensional networks stabilized by $\mathrm{H}$-bonds established between molecules; these interactions, in mixtures or suspensions of single polysaccharides, are disrupted when temperature increases. The results presented in Fig. 5 are in agreement with temperature stability experiments reported for galactomannans, that describe with temperature increase, a decrease in $G^{\prime}$, attributed to heat induced intra- and inter-chain $\mathrm{H}$-bonds breakage (Bourbon et al., 2010; Rinaudo \& Moroni, 2009; Shobha \& Tharanathan, 2009).

\section{Conclusions}

The extraction methodology described in this work proved adequate for obtaining galactomannan from $C$. grandis seeds, presenting a yield of $36 \pm 8 \%$ and a constant mannose/galactose ratio (2.44:1). Extensive experimental rheological characterization showed that the galactomannan presents a flow behavior, exhibiting Newtonian plateaus followed by shear-thinning zones up to $1.5 \%(\mathrm{w} / \mathrm{v})$ concentrations; above this value, its behavior can be considered as that of a polymer solution of entangled strands. At $2.0 \%$ concentration, rheological data indicate that the system has reached a gel-like state. The stress-strain study allowed a more accurate evaluation of this transition indicating $1.6 \%$ as the value for the sol-gel transition. The results show that the galactomannan studied here has appropriate characteristics for biotechnological applications in food and pharmaceutical industries.

\section{Acknowledgments}

The authors Priscilla B.S. Albuquerque and Gustavo R.C. Santos are recipient of a scholarship from Conselho Nacional de Desenvolvimento Científico e Tecnológico (CNPq). The authors express their gratitude to the Conselho Nacional de Desenvolvimento Científico e Tecnológico (CNPq) for research grants and fellowship (MTSC, PASM and MGCC). The authors acknowledge Centro de Tecnologias Estratégicas do Nordeste (CETENE) for analytical support and CNPq and Coordenação de Aperfeiçoamento de Pessoal de Nível Superior (CAPES) for financial support.

\section{References}

Andrade, C. T., Azero, E. G., Luciano, L., \& Gonçalves, M. P. (1999). Solution properties of the galactomannans extracted from the seeds of Caesalpinia pulcherrima and Cassia javanica: comparison with locust bean gum. International Journal of Biological Macromolecules, 26, 181-185.

Bose, S., \& Srivastava, H. C. (1978). Indian Chemical Society, 55, 1216-1218.

Bourbon, A. I., Pinheiro, A. C., Ribeiro, C., Miranda, C., Maia, J. M., Teixeira, J. A., et al. (2010). Characterization of galactomannans extracted from seeds of Gleditsia triacanthos and Sophora japonica through shear and extensional rheology: Comparison with guar gum and locust bean gum. Food Hydrocolloids, 24, 184-192.

Buckeridge, M. S., Panegassi, V. R., Rocha, D. C., \& Dietrich, S. M. C. (1995). Seed galactomannan in the classification and evolution of the leguminosae. Phytochemistry, 38(4), 871-875

Castelain, C., Doublier, J. L., \& Lefebvre, J. (1987). A study of the viscosity of cellulose derivatives in aqueous solutions. Carbohydrate Polymers, 7, 1-16.

Cerqueira, M. A., Lima, A. M., Souza, B. W. S., Teixeira, J. A., Moreira, R. A., \& Vicente, A. A. (2009). Functional polysaccharides as edible coatings for cheese. Journal of Agricultural and Food Chemistry, 57(4), 1456-1462.

Cerqueira, M. A., Pinheiro, A. C., Souza, B. W. S., Lima, A. M. P., Ribeiro, C., Miranda, C., et al. (2009). Extraction, purification and characterization of galactomannans from non-traditional sources. Carbohydrate Polymers, 75, 408-414.

Cerqueira, M. A., Bourbon, A. I., Pinheiro, A. C., Martins, J. T., Souza, B. W. S., Teixeira, J. A., et al. (2011). Galactomannans use in the development of edible films/coatings for food applications. Trends in Food Science E Technology, 22, 662-671.

Chaubey, M., \& Kapoor, V. P. (2001). Structure of a galactomannan from the seeds of Cassia angustifolia Vahl. Carbohydrate Research, 332(4), 439-444.

Ciucanu, J., \& Kerek, F. (1984). A simple and rapid method for the permethylation of carbohydrates. Carbohydrate Research, 131, 209-217.

Cross, M. M. (1965). Rheology of non-newtonian fluids: A new flow equation for pseudoplastic systems. Journal of Colloid Science, 20, 417.

Dakia, P. A., Wathelet, B., \& Paquot, M. (2007). Isolation and chemical evaluation of carob (Ceratonia siliqua L.) seed germ. Food Chemistry, 102, 1368-1374.

Da-Lozzo, E. J., Moledo, R. C. A., Faraco, C. D., Ortolani-Machado, C. F., Bresolin, T. M. B., \& Silveira, J. L. M. (2012). Curcumin/xanthan-galactomannan hydrogels: Rheological analysis and biocompatibility. Carbohydrate Polymers, 93(1), 279-284. 
Dea, I. C. M., \& Morrison, A. (1975). Chemistry and interactions of seed galactomannans. In R. S. Tipson, \& H. Derek (Eds.), Advances in Carbohydrate Chemistry and Biochemistry (pp. 241-312). New York: Academic Press.

Figueiró, S. D., Góes, J. C., Moreira, R. A., \& Sombra, A. S. B. (2004). On the physicochemical and dielectric properties of glutaraldehyde crosslinked galactomannan-collagen films. Carbohydrate Polymers, 56, 313-320.

Fringant, C., Tvaroska, I., Mazeau, K., Rinaudo, M., \& Desbrieres, J. (1995). Hydration of a-maltose and amylose: Molecular modelling and thermodynamics study. Carbohydrate Research, 278, 27-41.

Gidley, M. J., \& Reid, J. S. G. (2006). Galactomannans and other cell wall storage polysaccharides in seeds. In A. M. Stephen, G. O. Phillips, \& P. A. Williams (Eds.), Food polysaccharides and their applications. New York: CRC Press.

Graessley, W. W. (1967). Viscosity of entangling polydisperse polymers. The journal of Chemical Physics, 47, 1942-1953.

Jiang, J., Jian, H., Cristhian, C., Zhanga, W., \& Sun, R. (2011). Structural and thermal characterization of galactomannans from genus Gleditsia seeds as potential food gumsubstitutes. Journal of the Science of Food and Agriculture, 91, 732-737.

Joshi, H., \& Kapoor, V. P. (2003). Cassia grandis Linn f. seed galactomannan: structural and crystallographical studies. Carbohydrate Research, 338, 1907-1912.

Kapoor, V. P., Milas, M., Taravel, F. R., \& Rinaudo, M. (1996). Rheological properties of a seed galactomannan from Cassia siamea lamk. Food Hydrocolloids, 10, 167-172.

Kapoor, V. P., Taravel, F. R., Joseleau, J. P., Milas, M., Chanzy, H., \& Rinaudo, M. (1998). Cassia spectabilis DC seed galactomannan: structural crystallographical and rheological studies. Carbohydrate Reserach, 306, 231-241.

Kim, G. Y., Park, H. S., Nam, B. H., Lee, S. J., \& Lee, J. D. (2003). Purification and characterization of acidic proteo-heteroglycan from the fruiting body of Phellinus linteus (Berk \& M.A Curtis) Teng. Bioresource Technology, 89, 81-87.

Kircher, H. W. (1960). Gas-liquid chromatography of methylated sugars. Analyses Chemical, 32, 1103-1106.

Martins, J. T., Cerqueira, M. A., Souza, B. W. S., Avides, M. C., \& Vicente, A. A. (2010). Shelf life extension of ricotta cheese using coatings of galactomannans from nonconventional sources incorporating nisin against Listeria monocytogenes. Journal of Agricultural and Food Chemistry, 58(3), 1884-1891.

Martins, J. T., Cerqueira, M. A., Bourbon, A. I., Pinheiro, A. C., Souza, B. W. S., \& Vicente, A. A. (2012). Synergistic effects between k-carrageenan and locust bean gum on physicochemical properties of edible films made thereof. Food Hydrocolloids, 29, 280-289.

Mikkonen, K. S., Rita, H., Helen, H., Talja, R. A., Hyvõnen, L., \& Tenkanen, M. (2007). Effect of polysaccharide structure on mechanical and thermal properties of galactomannan-based films. Biomacromolecules, 8, 3198-3205.

Mudgil, D., Barak, S., \& Khatkar, B. S. (2012). Effect of enzymatic depolymerization on physicochemical and rheological properties of guar gum. Carbohydrate Polymers, $90,224-228$

Nwokocha, L. M., \& Williams, P. A. (2012). Rheological characterization of the galactomannan from Leucaena leucocephala seed. Carbohydrate polymers, 90, 833-838.

Peng, Y., Zhang, L., Zeng, F., \& Xu, Y. (2003). Structure and antitumor activity of extracellular polysaccharides from mycelium. Carbohydrate Polymers, 54, 297-303.

Perduca, M. J., Spotti, M. J., Santiago, L. G., Judis, M. A., Rubiolo, A. C., \& Carrara, C R. (2013). Rheological characterization of the hydrocolloid from Gleditsia amorphoides seeds. LWT-Food Science and Technology, 51, 143-147.
Pinheiro, A. C., Bourbon, A. I., Rocha, C., Ribeiro, C., Maia, J. M., Gonçalves, M. P., et al (2011). Rheological characterization of [kappa]-carrageenan/galactomannan and xanthan/galactomannan gels: comparison of galactomannans from nontraditional sources with conventional galactomannans. Carbohydrate Polymers, 83(2), 392-399.

Pollard, M. A., Kelly, R., Fischer, P. A., Windhab, E. J., Eder, B., \& Amadò, R. (2008) Investigation of molecular weight distribution of LBG galactomannan for flours prepared from individual seeds, mixtures and commercial samples. Food Hydrocolloids, 22, 1596-1606.

Pollard, M. A., Eder, B., Fischer, P., \& Windhab, E. J. (2010). Characterization of galactomannans isolated from legume endosperms of Caesalpinioideae and Faboideae subfamilies by multidetection aqueous SEC. Carbohydrate Polymers, 79, 70-84.

Rinaudo, M., \& Moroni, A. (2009). Rheological behavior of binary and ternary mixtures of polysaccharides in aqueous medium. Food Hydrocolloids, 23, 1720 1728.

Sandolo, C., Bulone, D., Mangione, M. R., Margheritelli, S., Di Meo, C., Alhaique, F., et al (2010). Synergistic interaction of Locust Bean Gum and xanthan investigated by rheology and light scattering. Carbohydrate Polymers, 82, 733-741.

Shobha, M. S., \& Tharanathan, R. N. (2009). Rheological behaviour of pullulanasetreated guar galactomannan on co-gelation with xanthan. Food Hydrocolloids 23, 749-754.

Shobha, M. S., Vishukumar, A. B., Tharanathan, R. N., \& Randgaonkar, A. K. (2005) Modification of guar galactomannan with the aid of Aspergillusniger pectinase. Carbohydrate Polymers, 62, 267-273.

Silveira, J. L. M., \& Bresolin, T. M. B. (2011). Pharmaceutical use of galactomannans. Química Nova, 34(2).

Srivastava, M., \& Kapoor, V. P. (2005). Seed galactomannans: An overview. Chemistry E' Biodiversity, 2, 295-317.

Tovar, A. M. F., Capillé, N. V. M., Santos, G. R. C., Vairo, B. C., Oliveira, S-N. M. C. G., Fonseca, R. J. C., et al. (2012). Heparin from bovine intestinal mucosa: Glycans with multiple sulfation patterns and anticoagulant effects. Thrombosis and Haemostasis, 107, 903-915.

Vendruscolo, C. W., Andreazza, I. F., Ganter, J. L. M. S., Ferrero, C., \& Bresolin, T. M. B (2005). Xanthan and galactomannan (from M. scabrella) matrix tablets for ora controlled delivery of theophylline. International Journal of Pharmaceutics, 296, $1-11$.

Vendruscolo, C. W., Ferrero, C., Pineda, E. A. G., Silveira, J. L. M., Freitas, R. A. Jimenez-Castellanos, M. R., et al. (2009). Physicochemical and mechanical characterization of galactomannan from Mimosa scabrella: effect of drying method. Carbohydrate Polymers, 76(1), 86-93.

Williams, P. A., \& Philips, G. O. (2000). Introduction to food hydrocolloids. In G. O. Phillips, \& P. A. Williams (Eds.), Handbook of hydrocolloids (2nd ed., pp. 1-19). New York: CRC Press.

Winter, H. H., \& Chambon, F. (1986). Analysis of linear viscoelasticity of a crosslinking polymer at the gel point. Journal of Rheology, 30, 367.

Yakimets, I., Paes, S. S., Wellner, N., Smith, A. C., Wilson, R. H., \& Mitchell, J. R. (2007) Effect of water content on the structural reorganization and elastic properties of biopolymer films: A comparative study. Biomacromolecules, 8, 1710-1722.

Yuen, S. N., Choi, S. M., Phillips, D. L., \& Ma, C. Y. (2009). Raman and FTIR spectroscopy study of carboxymethylated non-starch polysaccharides. Food Chemistry, 114, 1091-1098. 